\title{
Inverse Design of Built Environment by a Fast Fluid Dynamics-based Genetic Algorithm
}

\author{
Yu Xue ${ }^{1}$, Wei Liu ${ }^{2}$ \\ ${ }^{1}$ School of Civil Engineering, Dalian University of Technology, Dalian, China \\ ${ }^{2}$ Division of Sustainable Buildings, Department of Civil and Architectural Engineering, \\ KTH Royal Institute of Technology, Stockholm, Sweden
}

\begin{abstract}
It is essential to further design built environments with improved thermal comfort level, air quality, and reduced energy consumption of the HVAC system. Recently, researchers integrated computational fluid dynamics (CFD) with optimization algorithms to design the desired built environments. The CFD-based genetic algorithm, which is developed by imitating the evolution theory, is able to identify the global optima. However, the method has high requirements for the computational resources since a single design would take numbers of CFD simulations. Therefore, it is necessary to accelerate the CFD-based genetic algorithm, which would extend its application in the inverse design of built environment. A direct way to accelerate the CFD-based genetic algorithm is to find a substitute for the CFD simulations. The integration of fast fluid dynamics (FFD) and genetic algorithm seems to be able to accelerate the inverse design without losing the accuracy. Therefore, this investigation developed a FFD-based genetic algorithm and implemented the model in OpenFOAM (Open Field Operation and Manipulation), which is an open source CFD program. This study compared the FFD-based genetic algorithm with CFD-based genetic algorithm on accuracy and efficiency in the inverse design of thermal comfort and air quality in an office.
\end{abstract}

\section{Introduction}

A thermally comfortable, healthy, and productive built environment is typically achieved by the heating, ventilating, and air-conditioning (HVAC) system (McQuiston and Parker, 1982). On one hand, the HVAC system regulates the indoor air temperature and relative humidity within the thermal comfort zone (Standard, 2010). On the other hand, the HVAC system maintains healthy indoor air quality by supplying air that dilutes or removes the contaminants such as particles migrated from outdoor to indoor, VOC (volatile organic compounds), and infectious disease, etc (Spengler et al., 2001; Chen and Zhao, 2011). The HVAC system consumes tremendous amount of energy. For example, $41 \%$ of total en- ergy is used by buildings in U.S. and the energy used by HVAC systems accounts for about $50 \%$ of the total energy consumption (Administration et al., 2011). In China, the HVAC system consumes $20.7 \%$ of the total energy consumption (Cai et al., 2009). Even the HVAC system consumes such large amount of energy, there are multiple problems in the current built environments. For example, $42 \%$ percent of the occupants in U.S. are dissatisfied with the indoor thermal comfort level (Huizenga et al., 2006) and more than $40 \%$ of non-smoking adults and $60 \%$ of children aged 3-11 years old are exposed to tobacco smoke (US and Services, 2006). Besides, governments' requirement on the built environments is getting higher, especially in developing countries such as China (Bai et al., 2003). Therefore, it is essential to further design built environments with improved thermal comfort level, air quality, and reduced energy consumption of the HVAC system.

Recently, researchers integrated computational fluid dynamics (CFD) with optimization algorithms to design the desired built environments, because those methods are able to identify the optimal design variables(Liu et al., 2015). Those methods are proper orthogonal decomposition (POD) analysis, artificial neural network (ANN), CFD-based adjoint method, and CFD-based genetic algorithm (GA)(Chen et al., 2017). The first two methods could be regarded as substitutes for CFD simulations in the design procedure, but they need to first use CFD simulations as samples to establish or train the model. The POD analysis extracts the coherent structures of the air distributions into POD modes and provides a lowerorder description of the airflow by linear combinations of a small number of POD modes (Lumley, 1967). The ANN is trained by the samples and establishes the mapping relationship between the air distributions and the design variables (boundary conditions) in the design procedure (Zhou and Haghighat, 2009). The POD analysis and ANN could be much faster than the CFD simulations, but these two methods may have accuracy problems since the accuracy is highly dependent on the selection of the samples (Wei et al., 2016). The other two methods always 
use CFD simulation to obtain the air distributions and could be very accurate. The CFD-based adjoint method calculates the gradient of the objective function with respect to the design variables, so it can be very efficient. But this method is only able to identify the local optima and the design is highly dependent on the initial values for the design variables(Jameson, 1995). Further, since the CFD-based adjoint method requires complex mathematical manipulation and programming, its application in real engineering cases have high requirements for the designers (Liu et al., 2015). In contrast, the CFD-based GA is able to identify the global optima and the theory is developed by imitating the evolution theory, which is much more straightforward than that of the adjoint method. The CFD-based GA is more convenient for the designers to use. However, the method has high requirements for the computational resources since a single design would take numbers of CFD simulations. Liu et al. (2016) found that the CFD-based GA could be tens of times slower than the CFD-based adjoint method. Therefore, it is necessary to further accelerate the CFD-based GA, which would extend its application in the inverse design of built environment.

A direct way to accelerate the CFD-based GA is to find a substitute for the CFD simulations. For example, Li et al. (2013) integrated the POD analysis with GA to design the ventilation system in an office. Zhang and You (2014) integrated the ANN with GA for the inverse design of an aircraft cabin environment. Such integration would increase the design speed, but may have accuracy problems. To identify a trade-off between accuracy and efficiency, the fast fluid dynamics (FFD) that can solve the NavierStokes as accurately and informatively as does CFD simulation maybe a good option. FFD was first developed for atmospheric models (Staniforth and Côté, 1991) and further developed for simulating indoor airflow (Zuo and Chen, 2009). Recently, Liu et al. (2017) developed the FFD with turbulence models, which turned out to have the similar accuracy with CFD in simulating both steady-state and transient indoor airflow. The integration of FFD and GA seems to be able to accelerate the inverse design without losing the accuracy.

\section{Method}

The FFD-based GA applies the FFD simulations to obtain the fitness value to measure the quality of the designs. The fitness value is usually the value of a specified objective function for the design objective. This study used the thermal comfort level and indoor air quality as examples for the design objective. The corresponding objective function was constructed as:

$$
O(\boldsymbol{\xi})=\alpha \frac{\int_{\Omega}|P M V| d \Omega}{\int_{\Omega} d \Omega}+\beta \frac{\int_{\Psi} \tau d \Psi}{\int_{\Psi} d \Psi}
$$

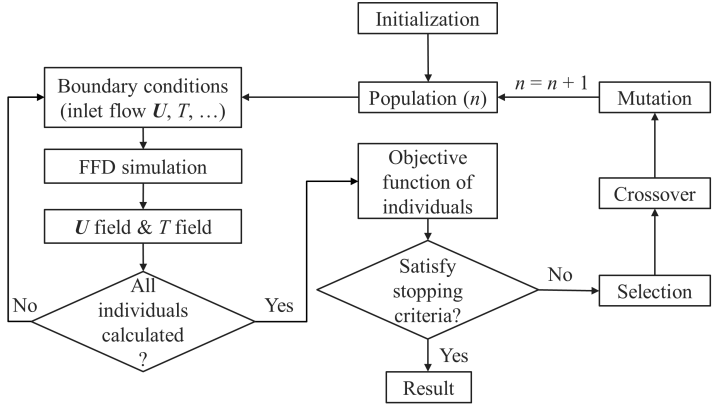

Figure 1: Flowchart of the FFD-based GA.

where $\boldsymbol{\xi}$ is a vector that represents the design variables (e.g., supply air velocity and temperature), $P M V$ is the predicted mean vote that used to evaluate the thermal comfort level, $\tau$ is the age of air that used to evaluate the air quality, $\Omega$ and $\Psi$ are the design domains, and $\alpha$ and $\beta$ are weighting factors. The objective function is constructed so that the design is to identify the $\boldsymbol{\xi}$ that minimizes the function. The following subsections introduce the principle and implementation in OpenFOAM for the GA and FFD one by one.

\section{Genetic Algorithm}

GA is a gradient-free optimization method. It imitates the evolution theory, uses operations such as selection, crossover, and mutation to generate new populations with smaller average objective functions (higher fitness values) Koza (1994). The smaller objective function an individual possesses, the closer it is to the optimal result. GA is capable for multivariable, multi-objective, and multi-solution problems, being able to find global optimumSakamoto et al. (1999). Figure 1 shows the FFD-based GA procedure that breaks down as follows:

1. Generate an initial population of potential solutions randomly, the population consist of $n$ individuals;

2. Evaluate the fitness value of each individual with specified objective function;

3. Check whether the population meets the prescribed optimization criterion; if not, apply evolution operations (selection, crossover, and mutation) to the individuals to generate a new population of potential solutions;

4. Repeat steps 2 and 3 until the optimization criterion is met.

For the evolution operations, GA firstly encodes the design variables of individuals into a chain of 01 codes that are similar to a chromosome. The design variables are encoded and managed independently. Then after the FFD simulations, individuals with smaller objective function would be selected to create a new population. This study applied the tournament-selection-method Deb (2000) that can distinguish individuals with similar objective functions. Then crossover occurs at a random posi- 
tion of the chain between two selected individuals. This study adopted a the only son crossover method that can always generate new individuals with smaller objective functionXue et al. (2013). At last a new individual is generated after mutation operation by switching 0 to 1 or 1 to 0 on the chain.

\section{Fast Fluid Dynamics}

The FFD in this study used the standard incremental pressure correction scheme Goda (1979) because its performance was slightly better than that of the other schemes Liu et al. (2017). This study considers the indoor airflow to be incompressible and viscous, then the Navier-Stokes equations to solve are:

$$
\begin{gathered}
\frac{\partial U_{i}}{\partial x_{i}}=0 \\
\frac{\partial U_{i}}{\partial t}+U_{j} \frac{\partial U_{i}}{\partial x_{j}}=-\frac{1}{\rho} \frac{\partial p}{\partial x_{i}}+\nu \frac{\partial^{2} U_{i}}{\partial x_{j} \partial x_{j}}+\frac{1}{\rho} F_{i} \\
\frac{\partial T}{\partial t}+U_{j} \frac{\partial T}{\partial x_{j}}=\kappa \frac{\partial^{2} T}{\partial x_{j} \partial x_{j}}+S_{T} \\
\frac{\partial \tau}{\partial t}+U_{j} \frac{\partial \tau}{\partial x_{j}}=\Gamma \frac{\partial^{2} \tau}{\partial x_{j} \partial x_{j}}+1
\end{gathered}
$$

where $U_{i}$ the $i_{t h}$ component of the velocity vector; $p$ the pressure, $\rho$ the density; $F_{i}$ the $i_{t h}$ component of the body forces; $\nu$ the effective viscosity; kappa the effective thermal conductivity; $S_{T}$ the energy source; and $\Gamma=\nu / 0.7$ the diffusion coefficient for age of air. FFD applies a two-step time-advancement scheme that splits the momentum equation (Eq. (3)) into two discretized equations:

$$
\begin{gathered}
\frac{U_{i}^{*}-U_{i}^{n}}{\Delta t}=-\frac{1}{\rho} \frac{\partial p^{n}}{\partial x_{i}}-U_{j}^{n} \frac{\partial U_{i}^{*}}{\partial x_{j}}+\nu \frac{\partial^{2} U_{i}^{*}}{\partial x_{j} \partial x_{j}}+\frac{1}{\rho} F_{i} \\
\frac{U_{i}^{n+1}-U_{i}^{*}}{\Delta t}=-\frac{1}{\rho} \frac{\partial\left(p^{n+1}-p^{n}\right)}{\partial x_{i}}
\end{gathered}
$$

where $U_{i}^{n}$ and $U_{i}^{n+1}$ represent the air velocity at the previous and current time steps, respectively; $U_{i}^{*}$ is the intermediate air velocity; $p^{n}$ and $p^{n+1}$ represent the air pressure at the previous and current time steps, respectively. Then FFD applies the pressure projection method (Chorin, 1968) that substitutes Eq. (7) into Eq. (2) to produce:

$$
\frac{\partial^{2}\left(p^{n+1}-p^{n}\right)}{\partial x_{i} \partial x_{i}}=\frac{\rho}{\Delta t} \frac{\partial U_{i}^{*}}{\partial x_{i}}
$$

FFD first solves Eq. (6) with the implicit Euler scheme for the temporal term; the semi-implicit scheme for the convection term; and the implicit scheme for the diffusion term to obtain intermediate

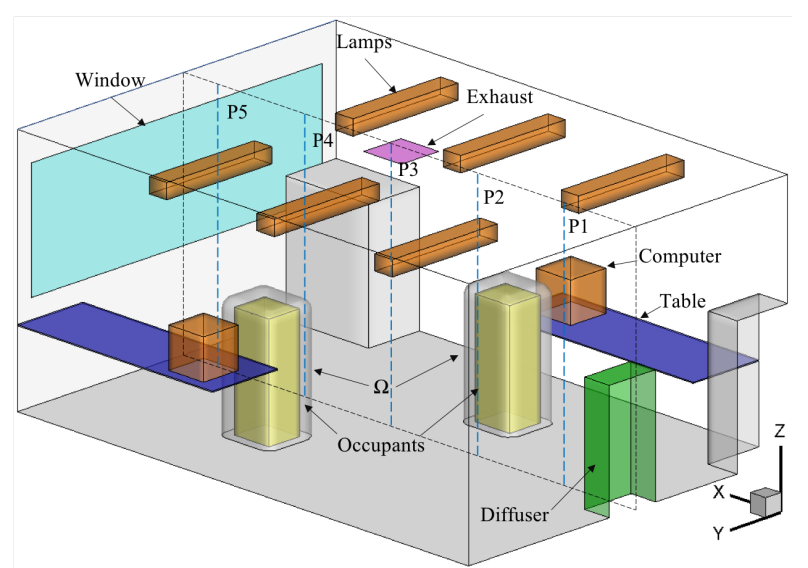

Figure 2: Schematic of an office with displacement ventilation.

velocity $U_{i}^{*}$. FFD then solves the Poisson equation, Eq. (8), to obtain the pressure $p^{n+1}$. Finally, the FFD calculates the air velocity at the next time step $U^{n+1}$ by solving Eq. (7) as follows:

$$
U_{i}^{n+1}=U_{i}^{*}-\frac{\Delta t}{\rho} \frac{\partial\left(p^{n+1}-p^{n}\right)}{\partial x_{i}}
$$

Eqs. (4) and (5) are linear partial differential equations, which are solved iteratively by using an implicit scheme. This study integrated FFD with the renormalization group (RNG) $k-\varepsilon$ model (Yakhot and Orszag, 1986) to simulate the turbulence because this model has been widely used for indoor airflow modeling (Zhang et al., 2007). The Boussinesq approximation (Boussinesq, 1903) was applied to simulate the buoyancy effect.

This study implemented the above mentioned FFD model in OpenFOAM that is a open source CFD toolbox with high level symbolic application programming interface.

\section{Results}

This study validated the FFD-based GA by comparing its performance with that of the CFD-based GA in designing the thermal environment in an office with displacement ventilation. The validation investigated the accuracy and efficiency by considering two aspects: the FFD in predicting the air distribution and the FFD-based GA in designing the desired built environments.

Figure 2 shows an occupied office with displacement ventilation. The office has the dimensions of $5.16 m \times 3.65 m \times 2.43 m$. Air was supplied from a displacement diffuser located on the side wall with a ventilation rate of four air changes per hour. The supply air temperature was $17^{\circ} \mathrm{C}$. There were two occupants, two computers, two tables, two boxes, and six lamps in the room. For detailed sizes, locations, and heat release of these items and other thermo-fluid boundary conditions, one can refer to Yuan et al. (1999). With all the boundary conditions from the 


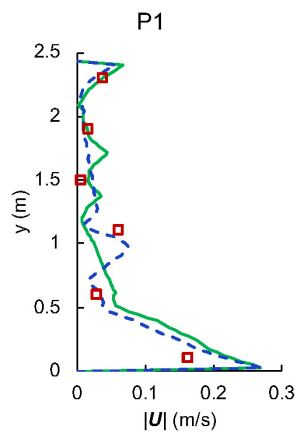

P1

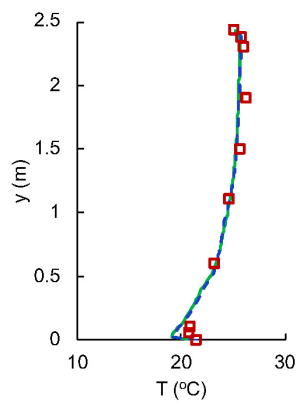

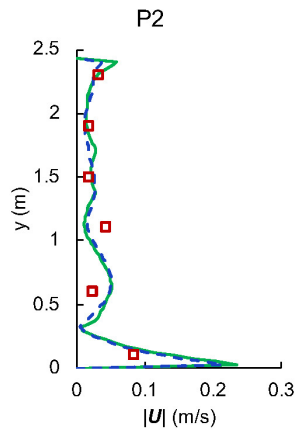

$\mathrm{P} 2$

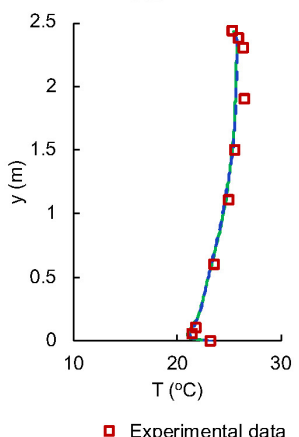

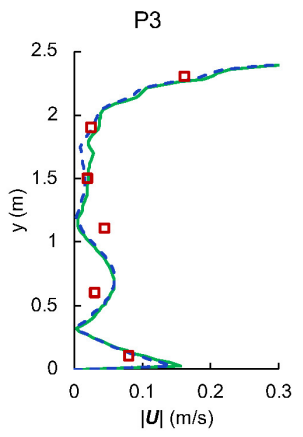

P3

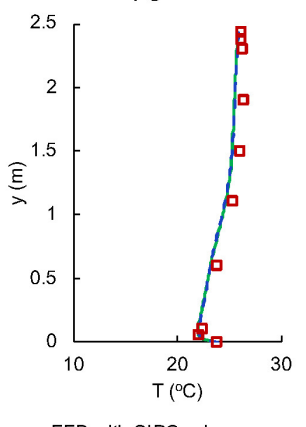

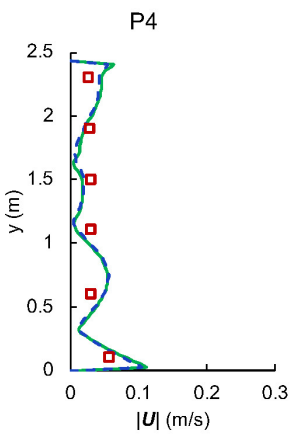

P4

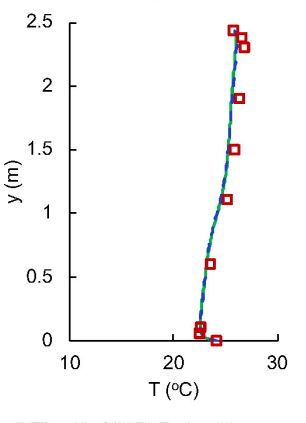

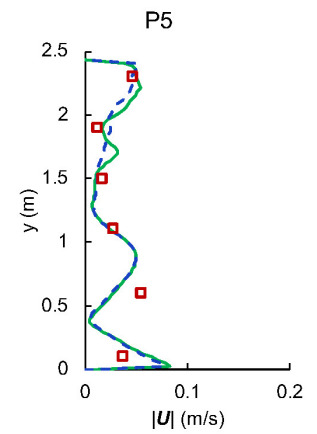

P5

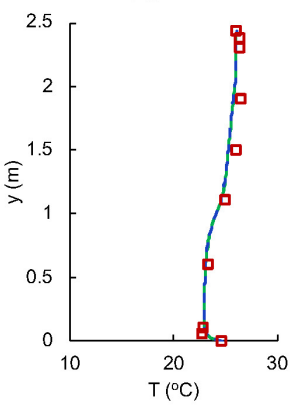

Figure 3: Comparison of the predicted air velocity and temperature profiles by FFD and CFD with the experimental data.

experiment, this investigation first conducted forward FFD and CFD simulations to confirm that the two methods lead to similar predictions. Figure 3 compares the predicted air velocity and temperature profiles with the experimental data on five vertical lines located in the $y$-directional center-cutting plane. One can refer to Figure 2 for the location of those vertical lines. Both predictions were almost the same and agreed well with experimental data.

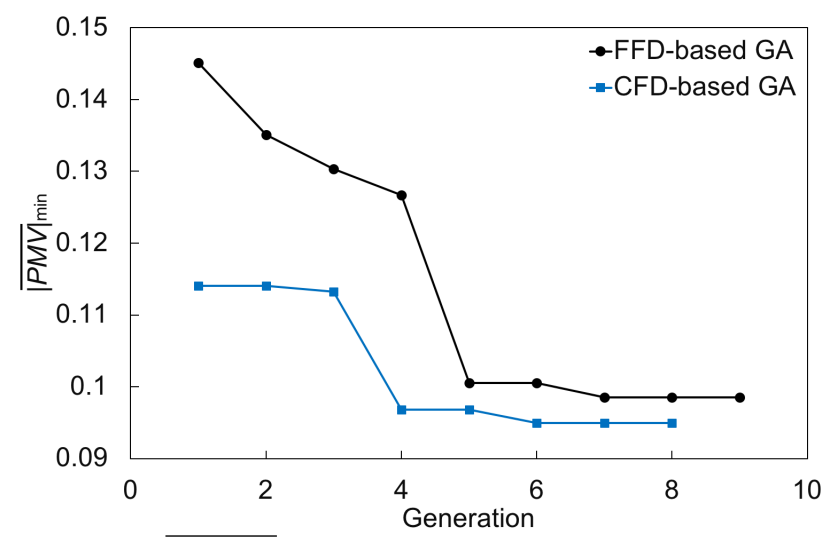

Figure 4: $\overline{|P M V|}_{\text {min }}$ of each generation for the FFDbased $G A$ and $C F D$-based $G A$.

Then this study applied the GA to design the air supply velocity magnitude $\left(|U|_{\text {inlet }} \in[0,5] \mathrm{m} / \mathrm{s}\right)$, velocity direction $\left(A_{\text {inlet }} \in\left[-25^{\circ}, 25^{\circ}\right]\right.$, the direction varied in the horizontal plane, where $0^{\circ}$ was the positive direction of $x$ axis and $25^{\circ}$ was the negative direction of $y$ axis $)$, and air temperature $\left(T_{\text {inlet }} \in[10,30]^{\circ} C\right)$. The design was to improve the thermal environment, so objective function was Eq. 1 with $\alpha=1$ and $\beta=0$. The surface $\Omega$ as shown in Figure 2 was assigned as the design domain. For the evolutionary process of GA, the investigation used eight individuals in a population and set the crossover rate value and mutation rate value as 0.8 and 0.1 , respectively. The convergence criteria was satisfied if the optimal result was repeated three times or the inverse design reached 100 generations.

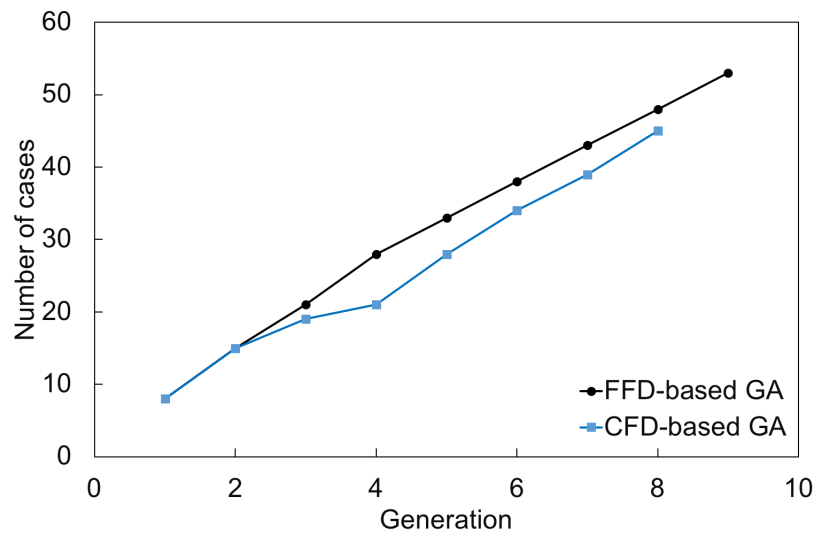

Figure 5: Number of cased calculated with respect to the generation for the FFD-based GA and CFD-based $G A$.

Figure 4 shows the $\overline{|P M V|}_{\text {min }}$ of each generation for inverse designs by FFD-based GA and CFD-based GA. Both of FFD-based GA and CFD-based GA tends to get smaller objective value along with the evolutionary process. FFD-based GA converged after 9 generations while CFD-based GA converged after 8 generations, which implied that the convergence speed of the two methods were similar. The optimal design of FFD-based GA was $\overline{|P M V|}_{\text {min }}=$ 0.09849 and that of CFD-based GA was $\overline{|P M V|}_{\text {min }}=$ 
Table 1: Design variables for the optimal designs by FFD-based GA and CFD-based GA.

\begin{tabular}{lllll}
\hline Methods & $|U|_{\text {inlet }}(\mathrm{m} / \mathrm{s})$ & $A_{\text {inlet }}\left({ }^{\circ}\right)$ & $T_{\text {inlet }}\left({ }^{\circ} \mathrm{C}\right)$ & $O(\boldsymbol{\xi})$ \\
\hline FFD-based GA & 0.14 & -4 & 23.5 & 0.9849 \\
CFD-based GA & 0.14 & -3.4 & 23.5 & 0.9498 \\
\hline
\end{tabular}

0.09498. The difference in the two designs were caused by the computing deviation between CFD and FFD, but the two designs were very close to each other according to the design variables for the optimal designs as summarized in Table 1.

As to the efficiency of the FFD-based GA, this study first compares the numbers of cases calculated by the two methods during the designs as shown in Figure 5. The number of cases calculated was proportional to the number of generations and the growth trends of both methods were similar, which means the numerical methods for solving the airflow had little influence on the optimization process of GA. However, the difference in computing time is significant between the two methods as shown in Figure 6. Benefit from the calculation speed of FFD, FFD-based GA can save more than $75 \%$ computation effort, as compared with CFD-based GA.

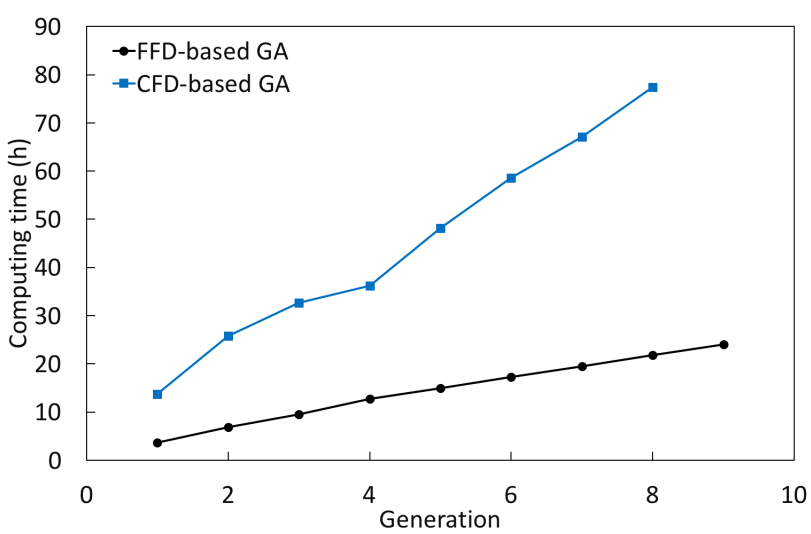

Figure 6: Computing time with respect to the generation for the FFD-based GA and CFD-based GA.

\section{Conclusion}

This study integrated FFD with genetic algorithm and implemented the FFD-based GA in OpenFOAM. This investigation validated the FFD-based GA by comparing its performance with that of the CFDbased GA in designing the thermal environment in an office with displacement ventilation. The validation found that the two designs were very close and the minor difference in the two designs were caused by the computing deviation between CFD and FFD. With similar design accuracies, FFD-based GA can save more than $75 \%$ computation effort, as compared with CFD-based GA.

\section{Acknowledgment}

This work was supported by the National Natural Science Foundation of China (Grant No. 51808487).

\section{References}

Administration, U. E. I. et al. (2011). Annual Energy Outlook 2011: With Projections to 2035. Government Printing Office.

Bai, Z., Z. Wang, T. Zhu, and J. J. Zhang (2003). Developing indoor air quality related standards in china. Journal of Asian Architecture and Building Engineering 2(1), 55-60.

Boussinesq, J. (1903). Théorie analytique de la chaleur: mise en harmonie avec la thermodynamique et avec la théorie mécanique de la lumière, Volume 2. Gauthier-Villars.

Cai, W., Y. Wu, Y. Zhong, and H. Ren (2009). China building energy consumption: situation, challenges and corresponding measures. Energy policy $37(6)$, 2054-2059.

Chen, C. and B. Zhao (2011). Review of relationship between indoor and outdoor particles: I/o ratio, infiltration factor and penetration factor. Atmospheric Environment 45(2), 275-288.

Chen, Q., Z. Zhai, X. You, and T. Zhang (2017). Inverse Design Methods for the Built Environment. Routledge.

Chorin, A. J. (1968). Numerical solution of the navier-stokes equations. Mathematics of computation 22(104), 745-762.

Deb, K. (2000). An efficient constraint handling method for genetic algorithms. Computer methods in applied mechanics and engineering 186(2-4), 311-338.

Goda, K. (1979). A multistep technique with implicit difference schemes for calculating two-or threedimensional cavity flows. Journal of Computational Physics 30(1), 76-95.

Huizenga, C., S. Abbaszadeh, L. Zagreus, and E. A. Arens (2006). Air quality and thermal comfort in office buildings: results of a large indoor environmental quality survey.

Jameson, A. (1995). Optimum aerodynamic design using cfd and control theory. In 12th Computational Fluid Dynamics Conference, pp. 1729.

Koza, J. R. (1994). Genetic programming as a means for programming computers by natural selection. Statistics and computing 4(2), 87-112. 
Li, K., W. Xue, C. Xu, and H. Su (2013). Optimization of ventilation system operation in office environment using pod model reduction and genetic algorithm. Energy and Buildings 67, 34-43.

Liu, W., R. Duan, C. Chen, C.-H. Lin, and Q. Chen (2015). Inverse design of the thermal environment in an airliner cabin by use of the cfd-based adjoint method. Energy and Buildings 104, 147-155.

Liu, W., R. You, Y. Xue, and Q. Chen (2016). Comparison and integration of generic algorithm and adjoint algorithm for the inverse design of indoor environment. In Proceedings of the 14th International Conference on Indoor Air Quality and Climate (Indoor Air 2016), Ghent, Belgium.

Liu, W., R. You, J. Zhang, and Q. Chen (2017). Development of a fast fluid dynamics-based adjoint method for the inverse design of indoor environments. Journal of Building Performance Simulation 10(3), 326-343.

Liu, W., T. Zhang, Y. Xue, Z. J. Zhai, J. Wang, Y. Wei, and Q. Chen (2015). State-of-the-art methods for inverse design of an enclosed environment. Building and Environment 91, 91-100.

Lumley, J. L. (1967). The structure of inhomogeneous turbulent flows. Atmospheric turbulence and radio wave propagation.

McQuiston, F. C. and J. D. Parker (1982). Heating, ventilating, and air conditioning: analysis and design.

Sakamoto, Y., A. Nagaiwa, S. Kobayasi, and T. Shinozaki (1999). An optimization method of district heating and cooling plant operation based on genetic algorithm. ASHRAE Transactions 105, 104.

Spengler, J. D., J. M. Samet, J. F. McCarthy, et al. (2001). Indoor air quality handbook.

Standard, A. (2010). 55 (2010). Thermal environmental conditions for human occupancy.

Staniforth, A. and J. Côté (1991). Semi-lagrangian integration schemes for atmospheric modelsa review. Monthly weather review 119(9), 2206-2223.

US, D. o. H. and H. Services (2006). The health consequences of involuntary exposure to tobacco smoke: a report of the surgeon general. Atlanta, GA: US Department of Health and Human Services, Centers for Disease Control and Prevention, Coordinating Center for Health Promotion, National Center for Chronic Disease Prevention and Health Promotion, Office on Smoking and Health 709.

Wei, Y., T. T. Zhang, and S. Wang (2016). Prompt design of the air-supply opening size for a commercial airplane based on the proper orthogonal decomposition of flows. Building and Environment 96, 131-141.

Xue, Y., Z. J. Zhai, and Q. Chen (2013). Inverse prediction and optimization of flow control conditions for confined spaces using a cfd-based genetic algorithm. Building and Environment 64, 77-84.

Yakhot, V. and S. A. Orszag (1986). Renormalization-group analysis of turbulence. Physical review letters 57(14), 1722.

Yuan, X., Q. Chen, L. R. Glicksman, Y. Hu, and X. Yang (1999). Measurements and computations of room airflow with displacement ventilation. Ashrae Transactions 105, 340-352.

Zhang, T.-H. and X.-Y. You (2014). Inverse design of aircraft cabin environment by coupling artificial neural network and genetic algorithm. HVACER Research 20(7), 836-843.

Zhang, Z., W. Zhang, Z. J. Zhai, and Q. Y. Chen (2007). Evaluation of various turbulence models in predicting airflow and turbulence in enclosed environments by cfd: Part 2comparison with experimental data from literature. Hvac\& $R$ Research 13(6), 871-886.

Zhou, L. and F. Haghighat (2009). Optimization of ventilation system design and operation in office environment, part i: Methodology. Building and Environment 44(4), 651-656.

Zuo, W. and Q. Chen (2009). Real-time or fasterthan-real-time simulation of airflow in buildings. Indoor air 19(1), 33-44. 